\title{
Avaliação de programas sociais e políticas públicas: panorama da produção científica de 1990 a 2015
}

O foco de especialistas e autoridades governamentais sob os resultados de programas sociais tem sido ampliado, cada vez mais torna-se importante para a Gestã Pública, que existam informações disponíveis sobre os programas desenvolvidos pelo Governo. O estudo apresenta conclusões de uma pesquisa a parti da revisão bibliométrica, debruçada sobre a literatura brasileira, nas duas últimas décadas, sobre o tema avaliação de programas sociais e políticas públicas. 0 objetivo foi apresentar a distribuição ao longo do período considerando localização geográfica da produção, temática, além dos principias centros de pesquisa e pesquisadores e suas relações. Uma investigação realizada de formada inovadora no Brasil, utilizando as bases do Google Scholar com a utilização do software Publish or Perish, tendo como descritores os termos "Avaliação de programas sociais" e "Avaliação de políticas públicas". Retornaram 329 textos dos quais 217 atenderam aos critérios da pesquisa. Os resultados cumpriram com os objetivos da pesquisa, retratando os principais autores, os temas relevantes, os periódicos, bem como os anos mais produtivos e os índices de impacto pelo Google Acadêmico. Observamos um crescimento das pesquisas no período, com prevalência dos temas Educação, Saúde, Segurança Alimentar, Agricultura e Emprego e Renda. Recomendamos a ampliação da discussão sobre a temática com uma proposta de agenda de pesquisa.

Palavras-chave: Gestão Pública; Avaliação; Pesquisa Bibliométrica; Políticas Públicas; Programas Sociais.

\section{Social programs and public policies: reviewed panorama of scientific production from 1990 to 2015}

The focus of experts and government officials under the results of social programs has been expanded, it becomes increasingly important for Public Management that there is information available on the programs developed by the Government. The study presents conclusions of a research on the bibliographic review, based on the Brazilian literature, about the last decades, in the evaluation of social programs and public policies. The objective was presented in a distribution throughout the period of construction of the production geography, thematic, in addition to research centers and researchers and their relationships. Research conducted using an innovative model in Brazil, using Google Scholar databases using the software Publish or Perish, using the terms "Evaluation of social programs" and "Evaluation of public policies" as descriptors. 329 results were found, of which 217 matches the search criteria. The results of the survey, depicting the main authors, relevant topics, periodicals and the most productive years, and Google Scholar impact indexes. We observed a growth of the research in the period, with prevalence of the disciplines Education, Health, Food Security, Agriculture and Employment and Income. We recommend an expanded discussion of a topic with the proposed research agenda.

Keywords: Public Administration; Evaluation, Bibliometric; Social Programs.

Topic: Gestão Pública

Reviewed anonymously in the process of blind peer.
Received: 12/10/2017

Approved: 14/12/2017
Gustavo Silva Menezes

Instituto Nacional de Tecnologia, Brasil http://lattes.cnpq.br/6184286169834097 gustavo.menezes09@gmail.com

Luciana Mourão

Universidade Salgado de Oliveira, Brasil http://lattes.cnpq.br/2558400549506524 mourao.luciana@gmail.com
Referencing this:

MENEZES, G. S.; MOURÃO, L.. Avaliação de programas sociais e políticas públicas: panorama da produção científica de 1990 a 2015. Revista Brasileira de Administração Científica, v.8, n.3, p.108-121, 2017. DOI: http://doi.org/10.6008/SPC2179-684X.2017.003.0009

DOI: 10.6008/SPC2179-684X.2017.003.0009 


\section{INTRODUÇÃO}

Tem aumentado o foco de especialistas e autoridades governamentais brasileiras sob indicadores de resultado de programas sociais. Além de apoiar a compreensão da dinâmica dos programas sociais implantados e em execução, os indicadores funcionam também como ferramenta para elaboração de novas políticas públicas, auxiliando na tomada de decisões (CAMPOS et al., 2011).

Assim, a demanda de políticos e gestores por indicadores que apresentem claramente as tendências do setor e os desdobramentos das políticas implantadas vem requerendo relatórios e manuscritos multidisciplinares voltados a análises métricas sobre a avaliação de programas sociais - APS. Tais avaliações compreendem, sobretudo, as áreas que realizam programas, o foco social atingido, o campo de atuação do proponente, os impactos identificados, e outras informações que permitem conhecer, melhorar e direcionar as políticas públicas continuamente (GASPARINI et al., 2014).

Apenas nas últimas décadas o Brasil inseriu na agenda pública a avaliação de políticas e programas governamentais, ao contrário de países como os Estados Unidos, Canadá, Espanha ou mesmo países latinoamericanos como o Chile e o México, que são mais experientes nas pesquisas dessa natureza (CANESQUI, 2015). Embora se tenha pouca tradição em avaliação de programas públicos no Brasil, o Tribunal de Contas da União, desde 1990, vem desenvolvendo trabalhos, sob a modalidade de auditorias operacionais, que tratam não somente da questão financeira dos programas públicos, mas também da avaliação de eficiência, economicidade e eficácia dos mesmos (HEDLER et al., 2009).

Outros fatos relevantes que contribuíram para os avanços na área da avaliação de programas e serviços sociais no País foram: (1) o advento da Política Nacional de Assistência Social (PNAS/2004), que ressalta a importância do monitoramento e avaliação de políticas sociais (GASPARINI et al., 2014); e (2) a criação da Política de Monitoramento e Avaliação do Ministério de Desenvolvimento Social e Combate à Fome (PMA/MDS) (Portaria n. 329, de outubro de 2006) e a criação da Secretaria de Avaliação e Gestão da Informação (SAGI) do MDS (DULCI, 2010).

De fato, na virada do século $X X$, o Brasil intensificou seus investimentos em políticas sociais, e as políticas de transferência de renda condicionadas ganharam particular relevância na agenda nacional (SANTOS, 2014; PEÑA, 2015), assim como as políticas públicas no campo habitacional (NASCIMENTO NETO, 2015). Porém, embora a avaliação de programas seja uma preocupação mais premente no Brasil nas últimas duas décadas, no final da década de 1970, Lima et al. (1978) já argumentava sobre a análise crítica de programas ou políticas públicas. Para Lima et al. (1978), o objetivo de tais avaliações era "apreender, principalmente, em que medida as metas estão sendo alcançadas, a que custo, quais os processos ou efeitos colaterais que estão sendo ativados (previstos ou não previstos, desejáveis ou não desejáveis) indicando novos cursos de ação mais eficazes" (LIMA et al., 1978).

Depreende-se a importância de investigar o que é produzido em termos de publicações relativas à área. Uma tendência que tem se observado em tais publicações é o crescimento de avaliações que discutem, não apenas um determinado programa, ou a metodologia de avaliação de programas de um modo geral, mas 
artigos metodológicos voltados para áreas específicas como a Educação (BAUER et al., 2015); a Saúde (VIEIRA-DA-SILVA, 2014); a Segurança Pública (MINAYO et al., 2005) dentre várias outras.

Essas produções permitem guiar futuras pesquisas no tema, além de permitir melhorias contínuas nos programas e políticas públicas. Assim, o presente estudo objetiva apresentar uma análise bibliométrica da produção científica nacional sobre o tema Avaliação de Programas Sociais nos últimos 25 anos. Esse tipo de estudo é elaborado a partir de material já publicado (GIL, 2002), constituído, neste caso, por artigos de periódicos disponibilizado na World Wide Web-WWW (internet) (RAVELLI et al., 2009).

\section{REVISÃO TEÓRICA}

\section{Estudo bibliométrico}

A bibliometria tem como objeto de estudo livros, artigos, documentos, revistas, autores e usuários (BUFREM et al., 2005; FERREIRA, 2010) e seu objetivo é informar sobre as fontes difusoras dos trabalhos, a evolução cronológica da produção científica, a produtividade de autores e instituições, o crescimento de determinado campo da ciência e o impacto das publicações frente à comunidade científica (REVELES et al., 2007). Essa metodologia tem sido usada multidisciplinarmente para apresentar relevantes informações sobre o desenvolvimento e comportamento da literatura de determinado assunto ao longo de uma época e, em um contexto determinado, buscando alinhamento para os registros do conhecimento por meio de um recurso quantificável (BUFREM et al., 2005; FERREIRA, 2010).

Para a realização de um estudo de natureza bibliométrica é importante conhecer o tema e delimitar bem o assunto pesquisado. Para além de uma análise numérica das publicações, busca-se transmitir a relevância do tema e o seu estudo na atualidade. Assim, apresentamos a seguir uma breve revisão da literatura sobre avaliação de programas sociais à luz de alguns dos principais autores do tema.

\section{Avaliação de Programas Sociais}

Figueiredo et al. (1986) define avaliação política como sendo outorgar valor às políticas, às suas consequências, ao aparato institucional em que elas se dão e aos próprios atos que pretendem modificar o conteúdo das mesmas No mesmo sentido, a avaliação de programas sociais, é aquela executada com base em políticas públicas e que, portanto, devem ter relações claras com a pesquisa de populações por amostragem, a análise de dados agregados (também chamados de contabilidade social), a análise de conteúdo e a observação do participante (ARRETCHE, 1998).

Aguilar et al. (1994), por sua vez, consideram que a avaliação é uma forma de pesquisa social aplicada, sistemática, planejada e dirigida; destinada a identificar, obter e proporcionar, de maneira válida e confiável, dados e informações suficientes e relevantes para apoiar um juízo sobre o mérito e o valor dos diferentes componentes de um programa. Essa definição é adotada no presente estudo devido a sua simultânea simplicidade e completude. Para Aguilar et al. (1994), a avaliação de programas pode ocorrer tanto na fase de diagnóstico, como na de planejamento e execução, permitindo comprovar a extensão e o grau em que se deram as conquistas, de forma a embasar ou guiar processos de tomada de decisão racionais 
entre cursos de ação, além de promover o conhecimento dos fatores associados ao êxito ou ao fracasso de seus resultados.

Arretche (1998) argumenta acerca da complexidade das avaliações de programas sociais. Inicialmente, é preciso identificar a variável relevante para o programa e seu verdadeiro intuito, separando os objetivos implícitos dos explícitos. Também cumpre analisar a dificuldade de se obter dados precisos e, muitas vezes, de entender seus resultados. Assim, torna-se útil nesse processo identificar as principais informações sobre o programa avaliado, buscando compreender suas características e singularidades.

Para Vedung et al. (1998), além da obtenção de conhecimentos básicos, a avaliação de programas também objetiva avaliar responsabilidade e melhorias obtidas. Chiechelski (2006), por sua vez, pondera que a avaliação de programas sociais tem sido realizada por dois tipos de processos: análise do avanço dos gastos públicos (encadeamento dos gastos sociais relativos ao gasto governamental total); e análise econômica (nível em que a utilização de recursos governamentais para a geração de bens e serviços e os resultados dos negócios sociais se coadunam com as necessidades previamente identificadas).

Assim, a APS permite mensurar o nível de atendimento da demanda em termos de metas físicas determinadas e do tipo de problema que o programa visa resolver (SOUZA, 2006). De um lado, essas avaliações podem ser de processo, no sentido de avaliar o ajustamento dos meios e recursos frente aos resultados parciais ou finais.

Do outro lado, a APS pode estar voltada ao impacto, compreendendo análises endereçadas aos efeitos do programa que podem ir além dos indivíduos participantes, compreendendo também resultados para grupos ou para a sociedade (FIGUEIREDO et al., 1986; VEDUNG et al., 1998; CARVALHO, 2003; CHIECHELSKI, 2006; SOUZA, 2006). A respeito desses níveis de diagnóstico, Carvalho (2003) reforça que a avaliação instrumental focaliza a análise da eficiência e da eficácia e, portanto, do processo da política ou programa, e que a avaliação de efetividade diz respeito aos impactos das ações promovidas pela política ou programa.

Assim, a APS inscreve-se no campo das Ciências Sociais Aplicadas, podendo beneficiar-se das abordagens da Microeconomia (com conceitos como os da maximização de benefícios e minimização de custos), da Ciência Política (apoiando-se na teoria dos conflitos e em conceitos como o da formação de arenas decisórias e das formas institucionais de governo), da Sociologia (considerando-se os referenciais conceituais para a elaboração de diagnósticos, reconstrução de processos sociais, etc.) (CARVALHO, 2003) ou da Psicologia (procedimentos científicos de pesquisa voltados para o comportamento humano (SECHREST, FIGUEIREDO, 1993; FERNÁNDEZ-BALLESTEROS et al., 1998) e para a desigualdade social (MCLEOD et al., 2014).

A respeito dessa preocupação com o rigor e com o método na realização das avaliações de programas sociais, é preciso considerar as argumentações apresentadas por autores como Oskamp (1984), Cronbach et al. (1982), Cook et al. (1986), Rossi et al. (1989), Posavac et al. (1997) ou autores mais atuais que estudam essa temática, a exemplo de Wholey et al. (2004), Worthen et al. (2004), Mourão et al. (2008), Oliveira et al. (2008), Bérubé et al. (2014), Planas et al. (2014) e Simmons (2015). 
Uma rápida análise da literatura nacional mostra que, apesar de serem recentes os estudos sobre APS, é crescente a quantidade de programas executados, experimentos realizados, autores e publicações sobre o tema. Por isso, é importante a realização de uma investigação mais aprofundada, promovendo estudos que apresentem uma análise da literatura científica. É relevante a apresentação de dados sobre os estudos das principais temáticas exploradas e as tendências e interesses prevalentes.

\section{METODOLOGIA}

Para a realização deste estudo bibliométrico, utilizamos as bases do Google Scholar (Google Acadêmico), tendo em vista as recentes indicações de abrangência e validade deste recurso (NORUZI, 2005; MUGNAINI et al., 2008; CRUZ, 2010; LUNKES et al., 2011; MARTINS, 2011). Tal base de dados permite acessar resumos de publicações situadas em outros repositórios científicos (Scielo, Sage, Periódicos Capes e outros). O modelo adotado pela presente pesquisa compreendeu uma fase inicial de seleção da base bibliográfica e uma fase subsequente de análise das categorias derivadas da classificação dos artigos. A figura 1 ilustra as estratégias de coleta definidas para a pesquisa.

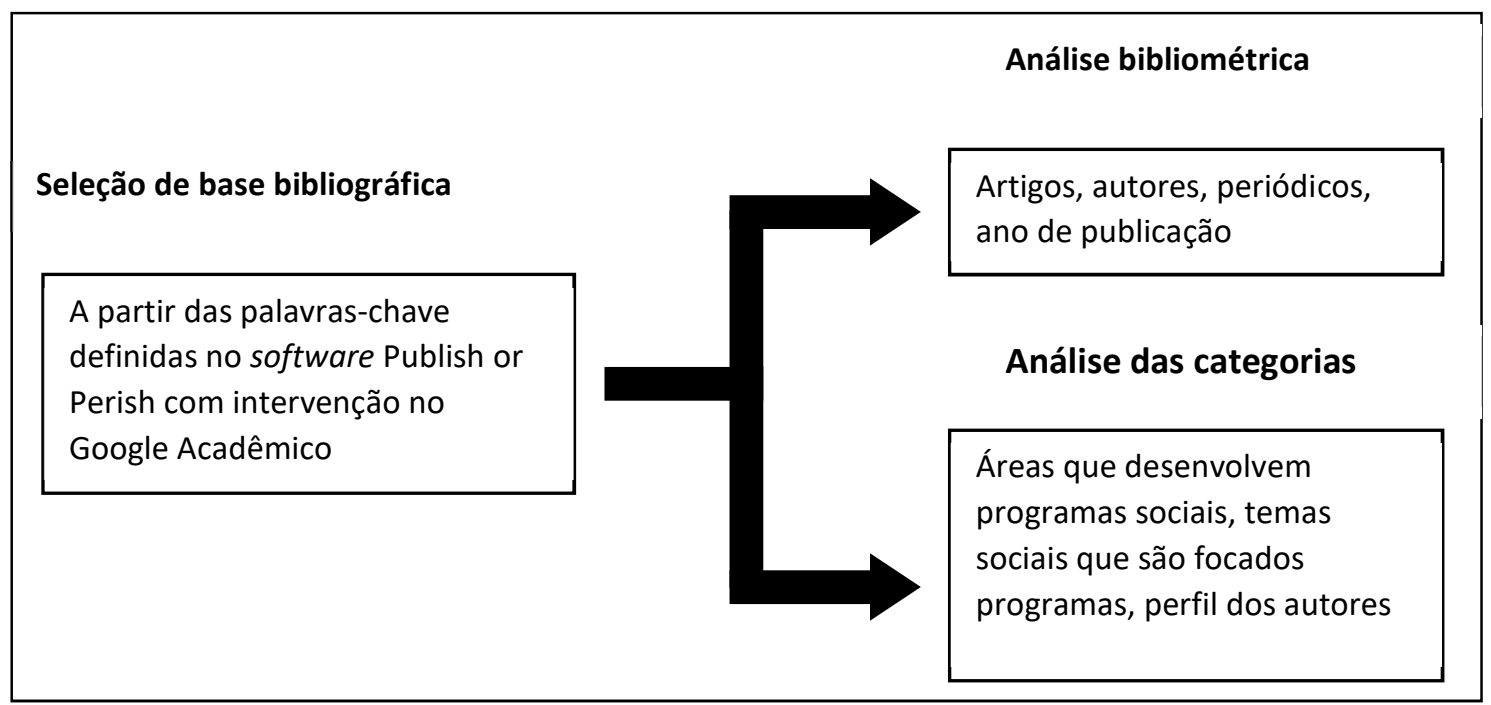

Figura 1: Estratégias de coleta do estudo bibliométrico.

\section{Amostra e procedimentos}

Os descritores considerados como argumentos significativos de busca (palavras-chave) para a pesquisa foram: Avaliação de programas sociais e Avaliação de políticas públicas. Como período de busca foi delimitando o início em 1990 e o fim em 2015, que correspondem aos últimos 25 anos e o período de maior produção no país sobre APS. Por fim, foi definido o critério do idioma português, tendo em vista que o interesse da revisão era na literatura nacional. Os argumentos foram pesquisados em separado com o uso de conectores booleano, que retornam os achados verdadeiros, porém com o conector ' $O R$ ' entre os descritores e 'AND' entre os seus termos, de forma que a expressão foi considerada somente quando aparecia de forma completa.

A busca foi realizada em agosto de 2016, com uma amostra de 449 textos retornados, com descarte de 8 textos repetidos. Foi considerada como condição de entrada a presença dos descritores, especificados, 
em artigos científicos no intervalo pesquisado. Como condição de saída foram observados artigos de língua portuguesa que não fossem do Brasil, os textos não considerados artigos científicos, a exemplo de programas de cursos de graduação e pós-graduação, pareceres sobre programas, livros e outros textos considerados fora do contexto da proposta do artigo. Assim, feitas as exclusões, com a utilização dos critérios de entrada e saída, foram eliminados 112 textos. Desse modo, a amostra do estudo foi composta por 337 artigos para análise (75\% dos textos retornados).

\section{Análise bibliométrica e de categorias}

Para a análise bibliométrica, foram utilizados os dados fornecidos pelo software Publish or Perish, que permite conhecer, de forma prática e ágil, as publicações no tema, o autor principal, o periódico, o ano e o índice de impacto da publicação. Esses dados foram transportados para uma planilha, para aplicação de filtros e ordenação, a fim de ampliar a quantidade de informações originadas.

Os objetivos dessa análise foram: observar o grau de relevância dos periódicos e dos autores; estimar o reconhecimento científico do tema; e conhecer a distribuição das publicações por período. Uma das contribuições dessa análise consistiu em permitir a criação de categorias por temática estudada, o que pode ser uma informação relevante para estudos futuros acerca do assunto.

Após a limpeza preliminar realizada no Excel, foi também desenvolvida uma análise categorial para relativa às principais áreas alcançadas pela APS e ao campo de atuação dos autores mais importantes. A partir da leitura prévia dos resumos de cada texto foi possível incluir essas informações na planilha. Após o término da composição, a mesma passou por uma fase de limpeza, em que foram aplicados filtros, para evitar informações duplicadas e também para permitir uma melhor qualidade e quantidade de informações geradas.

Identificamos e quantificamos quais temas formariam as categorias que têm recebido maior atenção por parte da APS, a saber: saúde, educação, segurança alimentar, agricultura, segurança, meio ambiente, esporte, trabalho e renda, habitação. Os textos que apresentaram vários temas ou que abordavam procedimentos gerais de avaliação foram categorizados, respectivamente, como Multitemáticos e Metodológicos. Dessa forma, a classificação buscou facilitar e clarear o entendimento sobre o tema e, ao mesmo tempo, minimizar distorções nos números pela inadequada contabilização de textos em mais de uma categoria. Contemplamos, ainda, uma análise da área dos autores de APS, uma vez que tanto a iniciativa privada, quanto as organizações não governamentais e o governo têm aparecido como operadores de avaliação.

\section{RESULTADOS}

A busca bibliométrica da produção científica sobre avaliação de programas entre 1990-2015 retornou textos de naturezas diferentes, entre elas: artigos, dissertações e teses válidos para a pesquisa pelo caráter informativo e acadêmico. Dessa forma, chamamos as publicações de 'textos', a fim de generalizar e, ao 
mesmo tempo facilitar a leitura do artigo sem a constante troca de termos, caso ficássemos especificando, a todo o tempo, a que tipo de manuscrito nos referimos.

Dos 337 textos que compõem a amostra identificamos diferentes temáticas. A área da Saúde destacou-se significativamente alcançando $17,5 \%$ do total, seguida pela área de Educação que teve 15,1\%. Na Figura 2, observamos que essas áreas foram acompanhadas por Emprego e Renda (10,1\%), Segurança Alimentar (8,3\%), Agricultura (5,3\%) e Habitação $(5,0 \%)$ e também áreas com menor expressão foram evidenciadas: Meio Ambiente (3,0\%), Segurança (1,2\%) e Esportes (0,9\%).

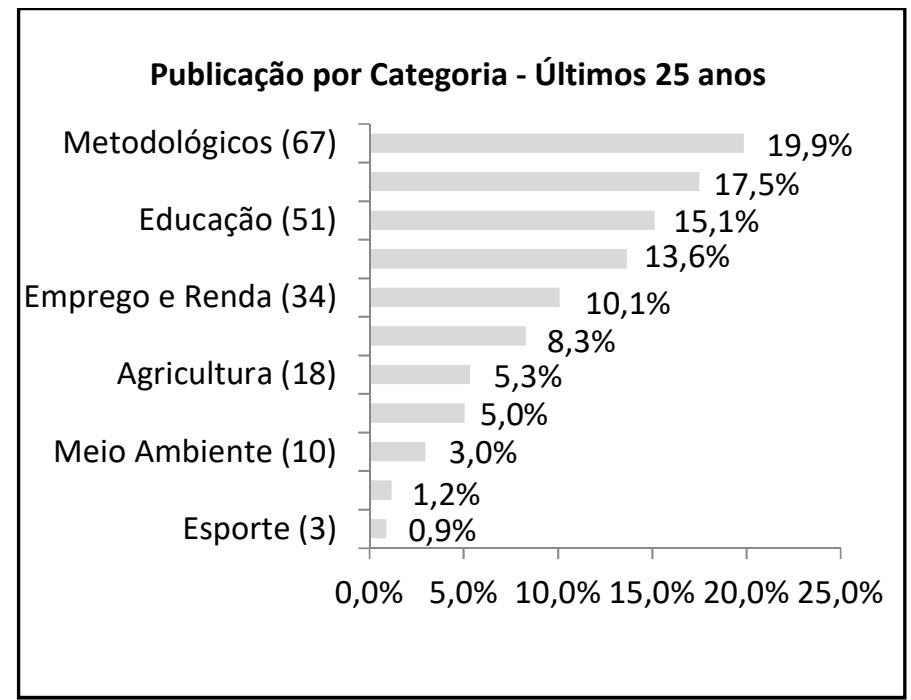

Figura 2: Distribuição de publicações por categoria.

Cabe explicar que duas grandes categorias concentram expressivos percentuais de recorrência: Metodológicos $(19,9 \%)$ e Multitemáticos $(13,6 \%)$. Esses grupos incorporam textos de diversas áreas do conhecimento, porém sua principal característica foi apresentar uma visão generalista da avaliação, quer seja por apresentar o processo de avaliação como ferramenta (Metodológicos), quer seja por abordar simultaneamente diversos temas relacionados ao bem-estar social, tais como Saúde, Educação, Renda, Habitação e Segurança (Multitemáticos). Os textos com essas características foram, a partir de então, analisados separadamente, para não criar uma tendência falaciosa nos números da pesquisa. Os demais textos foram categorizados conforme sua área temática.

Com relação ao ano das publicações, percebemos que, ao ano mais expressivo em números nominais, para o tema Avaliação de Programa ou Políticas Sociais foi 2014. Ele se destaca não só pela maior quantidade de publicação geral (49), como também para cada área separadamente, chegando a ter 13 textos publicados na categoria Saúde. Entretanto, Agricultura teve seu maior número de publicações em 2008 (6), enquanto Segurança Alimentar (6) e Meio Ambiente (3) tiveram seu auge de publicações em 2013.

Ressaltamos que, até o início do século XXI, era pequena a quantidade de publicações sobre avaliação de programas sociais, tendo o seu crescimento sido expressivo a partir de 2007, com 13 textos publicados, número que foi ampliado nos anos subsequentes. Esse resultado de aumento dos estudos sobre as políticas e programas públicos confirma discussões já realizadas por outros autores (SOUZA, 2006; NASCIMENTO NETO, 2015; PEÑA, 2015). 
$\mathrm{Na}$ análise das publicações por região (tabela 1), podemos perceber que, ao longo de todo o período, a região Nordeste (67) contou com maior número de produção por parte das universidades federais, a exemplo da Universidade Federal da Paraíba - UFPB, Universidade Federal de Pernambuco - UFPE, Universidade Federal do Rio Grande do Norte - UFRN, Universidade Federal do Maranhão - UFMA, Universidade Federal do Ceará - UFC e Universidade Federal da Bahia - UFBA. A região Sudeste vem em seguida com um número expressivo de publicações (61), porém algumas instituições particulares também compõem tal número. Seus principais representantes são: Fundação Getúlio Vargas - FGV, Pontifícia Universidade Católica - PUCSP, Universidade de São Paulo - USP, Universidade do Estado do Rio de Janeiro - UERJ, Universidade Federal de Viçosa - UFV, Universidade Federal de Lavras - UFLA, Universidade Federal do Rio de Janeiro - UFRJ.

Nas outras regiões do país a produção sobre avaliação de programas é bem menor. Na região Sul, os estudos aparecem em menor número (35), porém continuam seguindo a tendência de publicação por universidades com destaque para a Universidade Federal do Rio Grande do Sul - UFRGS, Universidade Federal de Santa Catarina - UFSC, Pontifícia Universidade Católica do Rio Grande do Sul - PUCRS e Universidade Federal do Paraná - UFPR. A região Centro-oeste totaliza 20 produções científicas, com estudos, principalmente, na Universidade de Brasília - UnB e Universidade Federal do Goiás - UFG. Por fim, foram encontradas poucas publicações atribuídas à região Norte do país, porém isso não necessariamente significa necessariamente uma ausência de avaliação na região, uma vez que estudos podem estar sendo realizados por pesquisadores com vínculos em instituições de outras regiões.

Tabela 1: Publicações por Universidades e Região.

\begin{tabular}{|c|c|c|}
\hline & UNIVERSIDADES RELEVANTES EM PUBLICAÇÕES & Total \\
\hline \multirow{5}{*}{ Nordeste } & Universidade Federal de Pernambuco - UFPE & 20 \\
\hline & Universidade Federal do Rio Grande no Norte - UFRN & 15 \\
\hline & Universidade Federal do Ceará - UFC & 13 \\
\hline & Universidade Federal da Paraíba - UFPB & 11 \\
\hline & Universidade Federal da Bahia - UFBA & 8 \\
\hline \multirow{7}{*}{ Sudeste } & Universidade Federal de Viçosa - UFV & 11 \\
\hline & Fundação Getúlio Vargas - FGV & 9 \\
\hline & Universidade de São Paulo - USP & 9 \\
\hline & Universidade Federal do Maranhão - UFMA (5) & 9 \\
\hline & Universidade do Estado do Rio de Janeiro - UERJ (5) & 5 \\
\hline & Universidade de São Carlos - UFSCAR & 5 \\
\hline & Universidade Federal do Rio de Janeiro - UFRJ & 5 \\
\hline & Universidade Estadual de Campinas - Unicamp & 5 \\
\hline & Pontifícia Universidade Católica - PUCSP & 3 \\
\hline \multirow{5}{*}{ Sul } & Universidade Federal do Rio Grande do Sul - UFRGS & 11 \\
\hline & Pontifícia Universidade Católica do Rio Grande do Sul - PUCRS & 9 \\
\hline & Universidade Federal de Minas Gerais - UFMG & 7 \\
\hline & Universidade Federal do Paraná - UFPR & 5 \\
\hline & Universidade Federal de Santa Catarina - UFSC & 3 \\
\hline \multirow{2}{*}{ Centro-Oeste } & Universidade de Brasília - UnB & 15 \\
\hline & Universidade Federal do Goiás - UFG & 5 \\
\hline
\end{tabular}

Além das universidades, outros atores - órgãos públicos, empresas do governo, institutos de pesquisa e fundações - mostram-se relevantes na produção de textos de avaliação de programas. Merecem destaque o Ministério do Desenvolvimento Social - MDS (35), Escola Nacional de Administração Pública Enap (19), o Instituto de Pesquisa Econômica Aplicada - Ipea (16), a Empresa Brasileira de Pesquisa Agropecuária - Embrapa (14), a Fundação João Pinheiro - FJP (9), e a Fundação Oswaldo Cruz - Fiocruz (6). 
Entre os periódicos, o destaque ficou para as seguintes revistas: Revista de Administração Pública RAP (16), Caderno de Saúde Pública (12), Cadernos de Pesquisa (7), Revista de Políticas Públicas (5), Saúde e Sociedade (4), Psicologia: Teoria e Pesquisa (3), Revista de Administração e Contabilidade (3) e Revista Brasileira de Educação Especial (3). Os 337 textos encontram-se publicados em 133 periódicos científicos e técnicos, sendo que os elencados acima responderam por $21 \%$ do total. O Scielo aparece como o principal repositório das publicações na internet, com mais de 50 textos apresentados, mas também foram encontradas produções diretamente em repositórios das universidades e de órgãos do governo.

$\mathrm{Na}$ tabela 2, apresentamos os textos mais citados ao longo dos últimos 25 anos, os autores, o ano de publicação, além do periódico e o repositório na internet, onde é possível encontrá-los. Observamos que a categoria de textos 'Metodológicos' recebe relevância por parte dos estudiosos que buscam referências para suas pesquisas e, também a categoria 'Saúde', ambas possuindo textos com os maiores números de citação absoluta.

Tabela 2: Textos mais citados no período.

\begin{tabular}{|c|c|c|c|c|c|}
\hline Citações & Autores & Títulos & Ano & Fonte & Repositório \\
\hline 152 & C. A. P. Faria & A política da avaliação de políticas públicas & 2005 & $\begin{array}{l}\text { Revista Brasileira de } \\
\text { Ciências Sociais }\end{array}$ & Scielo Brasil \\
\hline 148 & $\begin{array}{l}\text { K. Y. Uchimura, \& } \\
\text { M. L. M. Bosi }\end{array}$ & $\begin{array}{l}\text { Qualidade e subjetividade na avaliação de } \\
\text { programas e serviços em saúde }\end{array}$ & 2002 & $\begin{array}{l}\text { Cadernos de Saúde } \\
\text { Pública }\end{array}$ & ScieloPublic Health \\
\hline 112 & M. M. D. Cruz & $\begin{array}{l}\text { Avaliação de Políticas e Programas de saúde: } \\
\text { contribuições para o debate }\end{array}$ & 2011 & Repositório UERJ & ims.uerj.br \\
\hline 98 & P. D. M. Jannuzzi & $\begin{array}{l}\text { Considerações sobre o uso, mau uso e abuso dos } \\
\text { indicadores sociais na formulação e avaliação de } \\
\text { políticas públicas municipais }\end{array}$ & 2002 & $\begin{array}{l}\text { Revista de } \\
\text { Administração } \\
\text { Pública }\end{array}$ & bibliotecadigital.fgv.br \\
\hline 75 & $\begin{array}{l}\text { A. P., Trevisan \& } \\
\text { H. M.Van Bellen }\end{array}$ & $\begin{array}{c}\text { Avaliação de políticas públicas: uma revisão teórica } \\
\text { de um campo em construção }\end{array}$ & 2008 & $\begin{array}{l}\text { Revista de } \\
\text { Administração } \\
\text { Pública }\end{array}$ & Scielo Brasil \\
\hline
\end{tabular}

\section{DISCUSSÃO}

O estágio ainda pouco explorado do tema APS no Brasil, ajuda a compreender a ênfase em estudos sobre a ação de avaliar propriamente dita (metodológicos) ou em temas diversos (multitemáticos). Mas essa predominância das categorias macro (Metodológicos e Multitemáticos) tende a diminuir, inclusive com um movimento de artigos metodológicos por temática (MINAYO et al., 2005; VIEIRA-DA-SILVA, 2014; BAUER et al., 2015).

Isso também se confirma na análise das publicações (ano, autores, periódicos, região), em que é possível depreender que o crescimento das produções científicas sobre avaliação nas diversas categorias (Saúde, Educação, Emprego e Renda, Segurança Alimentar, Agricultura, Habitação, Meio Ambiente e Esporte), se faz em consonância com o crescimento do número de programas sociais estabelecidos nas mesmas. A análise mostra que a produção surge um tempo após a implementação de tais programas, por exemplo, o aumento do número de publicações na temática Segurança Alimentar provavelmente está associado à implantação do Programa de Aquisição de Alimentos - PAA, ocorrido em 2003. O mesmo é válido para os programas sociais relacionados ao Meio Ambiente e os Habitacionais.

Mas não é necessário que um programa tenha finalizado para que seja avaliado, pois a avaliação de programas pode ocorrer tanto na fase de diagnóstico, como na de planejamento e execução (AGUILAR et al., 1994). A avaliação não deve ser entendida ou confundida com a fase final do programa, a avaliação deve ser 
realizada ao longo de todo o processo, mesmo que focalize somente os efeitos relativos a fases anteriores (CARVALHO, 2003). Mas, cabe analisar que, as avaliações de processo são a priori de interesse dos gestores e, portanto, quando há resultados dos programas - ainda que parciais - é que a publicação de artigos se torna mais frequente.

Considerando-se que os últimos 15 anos concentram 90\% da produção dos últimos 25 anos, apresenta-se uma análise dos resultados ano a ano, conforme o tema foco da APS (tabela 3). Os resultados apontam que dados demográficos da população assistida por programas sociais no país influenciam na ênfase às categorias Saúde, Educação, Segurança Alimentar e Agricultura. Porém, nos últimos anos é possível observar o desenvolvimento de estudos sobre outras temáticas, possivelmente em decorrência do crescimento dos pesquisadores interessados nas mesmas. Ao longo dos anos $1990-1999$ foram encontrados 35 artigos distribuídos nos temas Saúde, Educação, Multitemáticos e Metodológicos, prioritariamente, os quais não estão apresentados na tabela 3, uma vez que este recorte objetiva mostrar como ficou a publicação nos últimos 15 anos.

Tabela 3: Produção de textos por categoria e ano de 2000 a 2015.

\begin{tabular}{|c|c|c|c|c|c|c|c|c|c|c|c|c|c|c|c|c|c|}
\hline Temas & 2000 & 2001 & 2002 & 2003 & 2004 & 2005 & 2006 & 2007 & 2008 & 2009 & 2010 & 2011 & 2012 & 2013 & 2014 & 2015 & Total \\
\hline Metodológicos & 3 & 2 & 2 & 2 & - & 3 & 4 & 2 & 3 & 5 & 4 & 8 & 6 & 7 & 7 & 5 & 63 \\
\hline Saúde & - & - & 1 & 1 & 2 & - & - & 1 & 3 & 2 & 1 & 5 & 5 & 5 & 13 & 10 & 49 \\
\hline Educação & 1 & - & - & - & - & - & 1 & 6 & 2 & 2 & 3 & 8 & 5 & 6 & 8 & 4 & 46 \\
\hline Multitemáticos & 2 & - & 2 & - & 1 & 1 & - & 1 & 3 & 3 & 6 & 4 & 6 & 4 & 5 & 4 & 42 \\
\hline $\begin{array}{l}\text { Emprego e } \\
\text { renda }\end{array}$ & 2 & - & - & - & - & - & - & - & 2 & 4 & 1 & 3 & 2 & 2 & 7 & 6 & 29 \\
\hline Seg. Alimentar & - & - & 1 & - & - & 1 & 1 & 1 & 1 & 2 & - & 3 & 2 & 6 & 4 & 3 & 25 \\
\hline Agricultura & - & 1 & - & 1 & - & - & - & - & 6 & 1 & 5 & 2 & 1 & - & 1 & - & 18 \\
\hline Habitação & - & - & - & - & 1 & - & - & - & - & - & 1 & 2 & - & 1 & 4 & 4 & 13 \\
\hline Meio Ambiente & 1 & - & - & - & - & - & - & 2 & - & 1 & - & 2 & 1 & 3 & - & - & 10 \\
\hline Segurança & - & - & - & - & - & 1 & & - & 1 & & - & 1 & - & 1 & - & - & 4 \\
\hline Esporte & - & - & - & - & - & - & - & - & - & - & 3 & - & - & - & - & - & 3 \\
\hline Total & 9 & 3 & 6 & 4 & 4 & 6 & 6 & 13 & 21 & 20 & 24 & 38 & 28 & 35 & 49 & 36 & 302 \\
\hline
\end{tabular}

Na tabela 4 destacamos o principal autor, a principal temática, a principal região, o ano em que houve mais publicação e o periódico que mais publicou nos últimos 25 anos. As colunas estão escalonadas de forma decrescente por mérito quantitativo, determinando a relevância das informações. Para que se compreendesse, denominou-se essa tabela de 'Principais informações sobre o artigo mais relevante nas quatro temáticas centrais da APS'.

Tabela 4: Principais informações sobre o artigo mais relevante nas quatro temáticas centrais da APS.

\begin{tabular}{|c|c|c|c|c|c|}
\hline Posição & Autor & Temática & Região & Ano & Periódico \\
\hline $\mathbf{1}^{\mathbf{a}}$ & Albuquerque F. J. B. & Saúde & Nordeste & 2014 & Revista de Administração Pública - RAP \\
\hline $\mathbf{2}^{\mathbf{a}}$ & Jannuzzi, P. M. & Educação & Sudeste & 2011 & Caderno de Saúde Pública \\
\hline $\mathbf{3}^{\underline{a}}$ & Rocha, F. E. C. & Emp. e Renda & Sul & 2015 & Revista de Políticas Públicas \\
\hline $\mathbf{4}^{\underline{a}}$ & Oliveira, S. F. & Seg. Alimentar & Centro-oeste & 2013 & Cadernos de Pesquisa \\
\hline
\end{tabular}

Como um aprofundamento do estudo bibliométrico, foram analisadas as dez publicações mais relevantes de APS nas temáticas de Saúde e Educação, tendo em conta o critério de citações. A leitura desses artigos permitiu observar que, na temática Saúde, os subtemas Saúde coletiva, Saúde da família e Sistema Único de Saúde - SUS tiveram maior representatividade, respondendo por seis dentre as dez publicações. Os subtemas gestão e trabalho em saúde, saúde do adolescente e avaliação de políticas de saúde completaram os artigos restantes. Fica também evidente a existência de uma preocupação em subáreas de maior 
abrangência populacional, com ênfase aos programas que têm maior amplitude, seja territorial ou populacional.

Os estudos em Educação deram ênfase aos programas direcionados a Educação Superior e Métodos de Avaliação das Políticas Educacionais, os quais correspondem a sete das dez principais publicações. A Educação Básica é o foco outras três publicações. Os estudos transparecem a ideia de que, com a entrada de novas políticas públicas (acesso ao ensino superior, auxílio financeiro para a graduação, ampliação do ensino técnico e profissional e bolsa para educação internacional), valorizou-se a formação de recursos humanos no país e o interesse em mensurar os resultados de tais programas. Já a atenção ao ensino básico volta-se, sobretudo, para a avaliação da qualidade da educação e quantidade disponível para atender a população.

Faz-se necessário refletir que, em meio às tensões sociais derivadas da crise do capitalismo nos anos 1970, surge uma corrente neoconservadora em contraposição ao Estado de bem-estar social. O neoconservadorismo combate o Estado social e os direitos sociais, opondo-se aos movimentos de defesa a minorias e almejando uma sociedade sem restrições ao mercado e em que o papel do Estado seja de reprimir as formas de contestação da ordem social (BARROCO, 2015).

No Brasil, o crescimento do chamado 'terceiro setor' ampliou as possibilidades de atuar em serviços sociais básicos, com mais programas sociais não apenas de responsabilidade governamental, mas também da sociedade civil organizada (YAMAMOTO, 2007). Além disso, a ampliação progressiva e sistemática das políticas públicas e ações do governo para atender a demandas sociais é um dos aspectos que contribuem para o aumento do quantitativo e relevância dos programas sócias, bem como das publicações acadêmicas sobre APS.

Por fim, após apresentadas as informações sobre a conjuntura econômica e as publicações, cabe nos relatar outro importante aspecto da pesquisa, que diz respeito à formação acadêmica e titulação dos autores mais frequentes e também dos autores dos artigos mais citados. Foram identificados profissionais de diferentes áreas do conhecimento propondo estudos e pesquisas relacionados à APS. Os autores em sua maioria absoluta (90\%) possuem titulação em nível de doutorado e os demais (10\%) em nível de mestrado. Se observarmos as áreas de formação dos principais autores, a Psicologia é a mais recorrente, embora haja um conjunto de outras áreas como Engenharia, Administração, Ciências Sociais, Matemática, Nutrição, História, Economia e Serviço Social.

Já com relação à titulação acadêmica em nível de doutorado, a Psicologia Social teve destaque, bem como, Ciências Sociais, Saúde Pública e Saúde Coletiva. Nas titulações em nível de mestrado, apenas Administração e Ciências Sociais foram observadas. Essas informações nos traduzem que o tema é explorado por diferentes áreas do saber que, contribuem para o desenvolvimento do tema e servem de referência para estudiosos que desejam realizar pesquisas focadas nas diferentes temáticas da APS.

\section{CONSIDERAÇÕES FINAIS}

A análise evolutiva das publicações permite constatar um crescimento na produção sobre APS, com destaque para as temáticas de Saúde e Educação. A despeito dessa evolução, observamos a necessidade da 
construção de indicadores e instrumentos que permitam uma análise comparada dos resultados produzidos nas diferentes áreas temáticas, uma vez que não se identificam instrumentos comuns nos estudos revisados. A presença de um maior número de textos nas categorias Metodológicos e Multitemáticos confirmam esse estágio inicial dos estudos brasileiros sobre APS, apontando a necessidade de se consolidar as bases temáticas.

Este estudo bibliométrico permitiu aumentar as informações sobre APS, suas tendências, temas de destaque, lacunas a serem exploradas, periódicos que dão ênfase, centros de estudo, regiões e pesquisadores mais relevantes. Nesse sentido, o panorama construído sobre a produção da área permite melhorar os estudos futuros, no sentido de sinalizar prioridades para investigações.

O momento político favorável ao avanço das políticas sociais, com crescimento de ações principalmente na região Nordeste fica evidenciado pelo fato de essa região concentrar a maioria dos estudos. Observa-se também que a região Sudeste, que tradicionalmente é uma das referências na produção de estudos de diferentes áreas, aparece com uma produção bastante expressiva. Não coincidentemente, o autor com maior número de publicações nas duas últimas décadas encontra-se na região Nordeste e pesquisa uma temática de importância crescente - a segurança alimentar.

Os resultados da análise dos resumos das publicações oriundas do estudo bibliométrico apontam para o desafio do desenho da avaliação. As definições do nível de resultado que deve ser pesquisado (eficiência, eficácia, efetividade ou impacto), da melhor forma de fazê-lo (ex-ante ou ex-post), do público a ser considerado (gestores, executores, participantes, etc.), do delineamento do estudo (correlacional ou experimental) podem se beneficiar da análise dos resultados de estudos anteriores.

Chama a atenção, por exemplo, que a qualidade de vida dos participantes - em última instância o objetivo principal de qualquer programa social - esteja pouco presente nos estudos de APS. Possivelmente, tal fato se deve à dificuldade de mensuração da qualidade de vida dos participantes de tais programas. Nesse sentido, como parte da agenda de pesquisa derivada do presente estudo, indica-se a criação de um inventário de qualidade de vida específico para a realidade brasileira, que considere as características particulares das políticas públicas, nas quais os programas se inserem.

O presente também apresenta limitações dignas de nota. A despeito de a amostra ter sido expressiva (337 estudos analisados), em buscas futuras devem ser usados mais e diferentes descritores, possibilitando a inserção de mais artigos à base da amostra. Além disso, a busca pode ser feita utilizando diferentes meta buscadores, uma vez que os resultados podem ser facilmente transportados para planilhas para exploração dos dados. Outra limitação do presente estudo é a apresentação de um panorama nacional, sem a discussão de especificidades regionais. Nesse sentido, cabe-nos sugerir estudos mais aprofundados sobre as publicações das diferentes regiões para o entendimento da evolução APS no país, tendo em vista suas diferenças regionais.

Concluímos ainda que a falta de informação completa sobre os autores nos artigos ou a indisponibilidade de currículo de muitos deles, traduz-se em uma das dificuldades da pesquisa bibliométrica. Evidencia-se, pois, a necessidade e importância de que os periódicos exijam a inclusão dos dados completos 
e uniformes dos autores, de modo a facilitar o desenvolvimento de estudos bibliométricos mais robustos e orientar melhor os leitores interessados em aprofundar as pesquisas nas temáticas publicadas.

Finalmente, para completar a agenda de pesquisa proposta para as pesquisas sobre APS no Brasil, sugere-se a realização de meta-análises e meta-sínteses, respectivamente para os estudos quantitativos e qualitativos de programas que já tenham uma quantidade relevante de avaliações realizadas, como é o caso dos programas Bolsa Família e Minha Casa Minha Vida. Esse tipo de pesquisa permitirá maior robustez às análises sobre os efeitos de tais programas, com maior possibilidade de contribuição para a redefinição de rumos dos mesmos ou de suas estratégias de implementação.

\section{REFERÊNCIAS}

ANDER-EGG, E.; AGUILAR, M. J.. Avaliação de serviços e programas sociais. Petrópolis: Vozes, 1994.

ARRETCHE, M. T. S.. Tendências no estudo sobre avaliação. Avaliação de Políticas Sociais, v.3, p.29-49, 1998.

BARROCO, M. L. S.. They will not pass! Neoconservative offensive and social work. Serviço Social \& Sociedade, n.124, p.623-636, 2015.

BAUER, A.; SOUSA, S. Z.. Indicadores para avaliação de programas educacionais: desafios metodológicos. Ensaio: Avaliação de Políticas Públicas Educacionais, Rio de Janeiro, v.23, n.86, p.259-284, 2015

BÉRUBÉ, A.; LAFANTAISIE, V.; DUBEAU, D.; CARON, J.; COUTU, S.; DEVAULT, A.. Using implementation evaluation to uncover a child neglect prevention program. Evaluation and Program Planning, v.45, p.1-8, 2014

BUFREM, L.; PRATES, Y.. O saber científico registrado e as práticas de mensuração da informação. Ciência da Informação, Brasília, v.34, n.2, p.9-25, 2005.

CAMPOS, R. T. O.; FURTADO, J. P.. Desafios da avaliação de programas e serviços de saúde. Campinas: Unicamp, 2011.

CANESQUI, A. M.. Avaliação de políticas e programas de saúde. Rio de Janeiro: Fiocruz, 2014.

CARVALHO, S. N.. Avaliação de Programas Sociais balanço das experiências. São Paulo em Perspectiva, v.17, n.4, p.185-197, 2003.

CHIECHELSKI, P. C. S.. Avaliação de programas sociais: abordagens quantitativas e suas limitações. Textos \& Contextos, Porto Alegre, v.4, n.1, p.1-12, 2006.

COOK, T. D.; SHADISH JUNIOR, W. R.. Program Evaluation: The Worldly Science. Annual Review of Psychology, v.37, n.1, p.193-232, 1986.

CRONBACH, L. J.; SHAPIRO, K.. Designing evaluations of educational and social programs. San Francisco: Josey-Bass, 1982.

CRUZ, D. C. M.. As vivências do cuidador informal do idoso dependente. Revista de Enfermagem Referência, Coimbra, v.2, p.127-136, 2010.
DULCl, O. S.. Avaliação de programas sociais: desafios e potenciais na construção de um sistema de informações. Cadernos de Estudos Desenvolvimento Social em Debate, Brasília, n.13, p.221-237, 2010.

FERNÁNDEZ-BALLESTEROS, R.; VEDUNG, E.; SEYFRIED, E.. Psychology in Program Evaluation. European Psychologist, Boston, v.3, n.2, p.143, 1998.

FERREIRA, A. G. C.. Bibliometria na avaliação de periódicos científicos. Data Grama Zero Revista de Ciência da Informação, v.11, n.3, p.1-9, 2010.

FIGUEIREDO, M. F.; FIGUEIREDO, A. M. C.. Avaliação política e avaliação de políticas: um quadro de referência teórica. 15 ed. São Paulo: IDESP, 1986.

GASPARINI, M. F. V.; FURTADO, J. P.. Avaliação de Programas e Serviços Sociais no Brasil: uma análise das práticas no contexto atual. Serviço Social \& Sociedade, São Paulo, n.117, p.122-141, 2014.

GIL, A. C.. Metodologia da pesquisa. São Paulo: Atlas, 2002.

HEDLER, H. C.; TORRES, C. V.. Meta-avaliação de auditorias de natureza operacional do tribunal de contas da união. Revista de Administração Contemporânea, Curitiba, v.13, n.3, p.469-486, 2009.

LIMA JUNIOR, O. B.; SILVA, A. A.; LEITE, M. C.. Intervenção planejada na realidade social: escopo e limites da pesquisa de avaliação. Rio de Janeiro: IPEA, 1978.

LUNKES, R. J.; FELIU, V. M. R.; ROSA, F. S.. Pesquisa sobre o orçamento na Espanha: um estudo bibliométrico das publicações em contabilidade. Revista Universo Contábil, Blumenau, v.7, n.3, p.112-132, 2011.

MARTINS, D.. A emergência da análise de redes sociais como campo de pesquisa: perspectiva da análise da produção científica em português e espanhol a partir do Google Acadêmico. Revista de Ciências de La Información, Lima, n.8, 2011.

MCLEOD, J. D.; SCHWALBE, M.; LAWLER, E. J.. Introduction. In: MCLEOD, J. D.; SCHWALBE, M.; LAWLER, E.. Handbook of the Social Psychology of Inequality. New York: Springer Dordrecht Heidelberg, 2014. 
MINAYO, M. C. S.; ASSIS, S. G.; SOUZA E. R.. Avaliação por triangulação de métodos: abordagem de programas sociais. Rio de Janeiro: Editora Fiocruz, 2005.

MOURÃO, L.; LAROS, J. A.. Avaliação de Programas Sociais: Comparando Estratégias de Análise de Dados. Psicologia: Teoria e Pesquisa, Brasília, v.24, n.4, p.545-558, 2008.

MUGNAINI, R.; STRECHL, L.. Recuperação e impacto da produção científica na era Google: uma análise comparativa entre o Google acadêmico e a Web of Science. Revista Eletrônica de Biblioteconomia e Ciência da Informação, Florianópolis, v.13, n.1, p.92-105, 2008.

MUKHERJEE, B.. Journal of the American Society for Information Science and Technology (2000-2007): a bibliometric study. International Federation of Library Associations and Institutions: IFLA Journal, Amsterdã, v.35, n.4, p.341-358, 2009.

NASCIMENTO NETO, P.. Análise de políticas públicas: entre a modelagem e a realidade da política habitacional brasileira. Revista de Administração Pública, Rio de Janeiro, v.49, n.4, p.847-868, 2015.

NORUZI, A.. Google Scholar: The new generation of citation indexes. Libri, v.55, n.4, p.170-180, 2005.

OSKAMP, S.. Applied Social Psychology. Washington: Prentice-Hall, 1984.

PEÑA, C. R.. A eficácia das transferências de renda: as tendências da desigualdade antes e depois do Programa Bolsa Família. Revista de Administração Pública, Rio de Janeiro, v.49, n.4, p.889-913, 2015.

PLANAS, A.; VILÀ, P. S. M.. Assessing youth policies: a system of indicators for local government. Evaluation and Program Planning, v.45, p.22-28, 2014.

POSAVAC, E. J.; CAREY, R. G.. Program Evaluation: methods and case studies. New Jersey: Prentice-Hall, 1997.

PRITCHARD, A.. Statistical bibliography or bibliometrics?. Journal of Documentation, n.25, p.348-349, 1969.

RAVELLI, A. P. X.; FERNANDES, G. C. M.; BARBOSA, S. F. F.; SIMÃO, E.; SANTOS, S. M. A.; MEIRELLES, B. H. S.. A produção do conhecimento em enfermagem e envelhecimento: estudo bibliométrico. Texto \& Contexto Enfermagem, Florianópolis, v.18, n.3, p.506, 2009.
REVELES, A. G.; TAKAHASHI, R. T.. Educação em saúde ao ostomizado: um estudo bibliométrico. Revista Escola de Enfermagem USP, São Paulo, v.41, n.2, p.245-50, 2007.

SANTOS, M. C. M.. A voz do beneficiário: uma análise da eficácia do Programa Bolsa Família. Revista de Administração Pública, v.48, n.6, p.1381-1405, 2014.

SECHREST, L.; FIGUEIREDO, A. J.. Program Evaluation. Annual Review of Psychology, v.44, n.1, p.645-674, 1993.

SIMMONS, V. N.. Participatory evaluation of a communityacademic partnership to inform capacity-building and sustainability. Evaluation and Program Planning, v.52, p.1926, 2015.

SOUZA, C.. Políticas públicas: uma revisão da literatura. Sociologias, Porto Alegre, v.8, n.16, p.20-45, 2006.

TREVISAN, A. P.; BELLEN, H. M.. Avaliação de políticas públicas: uma revisão teórica de um campo em construção. Revista de Administração Pública, Rio de Janeiro, v.42, n.3, p.529-550, 2008.

VANTI, N. A. P.. Da bibliometria à webometria: uma exploração conceitual dos mecanismos utilizados para medir o registro da informação e a difusão do conhecimento. Ciência da Informação, Brasília, v.31, n.2, p.152-162, 2002.

VEDUNG, E.; BEMELMANS-VIDEC, M. L.; RIST, R. C.. Policy instruments: typologies and theories: carrots, sticks, and sermons. Policy instruments and their evaluation, v.5, p.21$58,1998$.

VIEIRA-DA-SILVA, L. M.. Avaliação de políticas e programas de saúde. Rio de Janeiro: Editora Fiocruz, 2014.

WHOLEY, J. S.; HATRY, H. P.; NEWCOMER, K. E.. Handbook of practical program evaluation. Nova Jersey: John Wiley \& Sons, 2010.

WORTHEN, B. R.; SANDERS, J. R.; FITZPATRICK, J. L.. Avaliação de programas: concepções e práticas. São Paulo: EDUSP, 2004

YAMAMOTO, O. H.. Políticas sociais, 'terceiro setor' e 'compromisso social': perspectivas e limites do trabalho do psicólogo. Psicologia \& Sociedade, v.19, n.1, p.30-37, 2007. 